\title{
Functional characterization of the active Mutator-like transposable element, Muta 1 from the mosquito Aedes aegypti
}

\author{
Kun Liu ${ }^{1}$ and Susan R. Wessler ${ }^{2^{*}}$
}

\begin{abstract}
Background: Mutator-like transposable elements (MULEs) are widespread with members in fungi, plants, and animals. Most of the research on the MULE superfamily has focused on plant MULEs where they were discovered and where some are extremely active and have significant impact on genome structure. The maize MuDR element has been widely used as a tool for both forward and reverse genetic studies because of its high transposition rate and preference for targeting genic regions. However, despite being widespread, only a few active MULEs have been identified, and only one, the rice Os3378, has demonstrated activity in a non-host organism.
\end{abstract}

Results: Here we report the identification of potentially active MULEs in the mosquito Aedes aegypti. We demonstrate that one of these, Muta1, is capable of excision and reinsertion in a yeast transposition assay. Element reinsertion generated either 8 bp or 9 bp target site duplications (TSDs) with no apparent sequence preference. Mutagenesis analysis of donor site TSDs in the yeast assay indicates that their presence is important for precise excision and enhanced transposition. Site directed mutagenesis of the putative DDE catalytic motif and other conserved residues in the transposase protein abolished transposition activity.

Conclusions: Collectively, our data indicates that the Muta1 transposase of Ae. aegypti can efficiently catalyze both excision and reinsertion reactions in yeast. Mutagenesis analysis reveals that several conserved amino acids, including the DDE triad, play important roles in transposase function. In addition, donor site TSD also impacts the transposition of Muta1.

Keywords: Transposable elements, Mutator-like elements (MULE), Aedes aegypti, Yeast assay, Target site duplication (TSD), Transposase

\section{Background}

Transposable elements (TEs) are mobile fragments of DNA that can move from one locus to another in the host genome, often replicating in the process. TEs usually make up the largest fraction of eukaryotic genomes; accounting for almost half of the human genome, and more than $70 \%$ of the genomes of some grass species [1]. Based on their transposition intermediate, eukaryotic TEs are divided into two classes. Class 1 elements utilize an RNA intermediate in the transposition reaction while the intermediate for most class 2 elements is the element itself that is mobilized by a 'cut and paste' mechanism [2].

\footnotetext{
* Correspondence: susan.wessler@ucr.edu

${ }^{2}$ Department of Botany and Plant Sciences, University of California, Riverside, CA 92521, USA

Full list of author information is available at the end of the article
}

A TE family is composed of one or more transposaseencoding autonomous elements and up to several thousand nonautonomous elements that do not encode functional transposase. Family members share the same terminal inverted repeats (TIRs) and target site duplications (TSDs) of the same length allowing them to move in trans by utilizing the transposase encoded by the autonomous family member(s) [3].

Prior studies have classified class 2 TEs into upwards of 19 superfamilies on the basis of the relatedness of the element-encoded transposase [4]. The original Mutator element, now called $M u D R$, was first isolated from a maize strain as the agent responsible for its high forward mutation rate $[5,6]$. Subsequently, members of this superfamily, called collectively Mutator-like transposable elements (MULEs), were found in other plants, and in 
fungi, protozoans, and in a variety of animals, (from insects to fish to other metazoans) [3, 7-9]. Typical features of MULEs include long terminal inverted repeats (TIRs) (>100 bp) and an 8-10 bp TSD [10]. Nonautonomous family members often contain a variety of sequences between the TIRs, including fragments from host genes; such elements are called Pack-MULEs [11]. To date, only a few active MULEs have been identified, including MuDR, Hop1, Jittery, TED, and Os3378 [6, 12-15]. Importantly, only the rice Os3378 element has been shown to transpose in a heterologous host [15].

Most MULE transposases contain a $\mathrm{N}$-terminal zinc finger DNA binding motif and a conserved C-terminal DDE domain, which has been shown to be the catalytic core for transposition reactions in other superfamilies but not to date for MULEs [16, 17]. Phylogenetic studies indicate that the DDE domain of MULE transposases is closely related to the IS256 family, which is present in diverse prokaryotes [18]. Each residue of the predicted DDE motif of IS256 has been shown to be necessary for transposition [19]. Most MULE transposases also harbor a $\mathrm{CH}$ motif and a $\mathrm{W}$ residue between the second $\mathrm{D}$ and $\mathrm{E}$ of the DDE domain, which are also conserved in the $h A T$ superfamily. Mutagenesis analyses in $h A T$ elements demonstrated the importance of these residues for transposition [20, 21]. Whether these residues are also important for the MULE transposase has not as yet been determined.

TE-based genetic tools have facilitated our deep understanding of the biology of both plants and animals, however, such tools are not currently available in the mosquito species, including Aedes aegypti, Aedes albopictus, Culex quinquefasciatus and Anopheles gambiae, which can spread dengue fever, yellow fever, chikungunya, zika, and many other diseases that are responsible for over one million deaths annually [22-25]. Four exogenous TEs that transpose at high frequencies in Drosophila melanogaster (Hermes, Mos1, Minos, piggyBac) were found to move rarely or not at all in the germ line of mosquitoes as very few germinal mutations were detected [26, 27]. To explain this result, it was hypothesized that mosquitoes have a strong genome defense system that could effectively recognize and silence foreign TEs [28]. Therefore endogenous active TEs could be effective mutagens from generation to generation as they might be able to evade genome surveillance. Availability of the genome sequence enabled the search for potentially active TEs in the mosquito genomes [29-32]. In this study we show that Muta1, identified by computer-assisted analysis of the Ae. aegypti genome, is an active TE capable of both insertion and excision in a yeast transposition assay. Site-directed mutagenesis analysis revealed that transposition activity in yeast was influenced by disruption of several conserved residues and by the presence of TSDs at the donor site. With characteristics such as high transposition activity, precise excision, and no target sequence preference, Muta1 could be crafted into an effective tool for forward mutation analyses in mosquitoes.

\section{Results}

Distribution of the MULE superfamily in mosquito genomes To identify potentially active MULEs, we first assessed the abundance and distribution of MULEs in the genomes of Ae. aegtpti, Ae albopictus, C quinquefasciatus, and $A n$. gambiae [29-32]. The conserved MULE DDE domain sequences were used as queries in TBlastN searches against the Ae. aegypti genome through the TARGeT program, which is designed for TE discovery [20, 33]. After removal of duplicate hits, sequences with significant similarity (e- value $<10^{-15}$ ) to the MULE DDE domain were identified and used to build a phylogenetic tree. As a result, no significant hits were identified in the An. gambiae genome, whereas 141, 105, and 10 putative MULE DDE domain sequences were identified from the Ae. aegtpti, Ae albopictus, and $C$ quinquefasciatus genomes, respectively (Additional file 1: Figure S1).

Full-length elements were defined by comparison of sequences in the same branch of the phylogenetic tree where adjacent sequences share high similarity that extends beyond the DDE domain. Sequences near the boundary of similarity were examined manually for the long TIRs and 8-10 bp TSDs that are features of MULEs. The 10 hits in C. quinquefasciatus were likely remnants of ancient insertion events as they are highly divergent in sequence and lack identifiable TIR and TSD. The quality of the Ae. albopictus genome assembly prevented our search for full-length elements. In the Ae. aegypti genome, 31 full-length elements sorted into 14 families, each with $>95 \%$ sequence similarity (Muta1-14, Fig. 1).

\section{Characterization of the MULE superfamily in Ae. aegypti}

All of the 14 MULE families identified from Ae. aegypti have $8-9$ bp TSD, 12 of 14 have TIRs $>100 \mathrm{bp}$ and two have TIRs $<60 \mathrm{bp}$. Ten of the 12 long TIR families contain short subterminal tandem repeats $(9-15 \mathrm{bp})$. All families except Muta6 also contain derivative nonautonomous elements (Additional file 2: Table S1), which share high sequence similarity in their TIR and subterminal regions, but carry heterogeneous internal sequences including fragments of host genes.

The phylogenetic tree also reveals the evolutionary relationships of the 14 families. Among the 5 major groups resolved on the tree, group $B$ includes half of the 14 families, while group D and E contains one family each (Fig. 1). Furthermore, long branches indicate extensive sequence differences between families. For example, although Muta5 and Muta8 belong to group A and locate to adjacent clades, no significant nucleotide similarity 


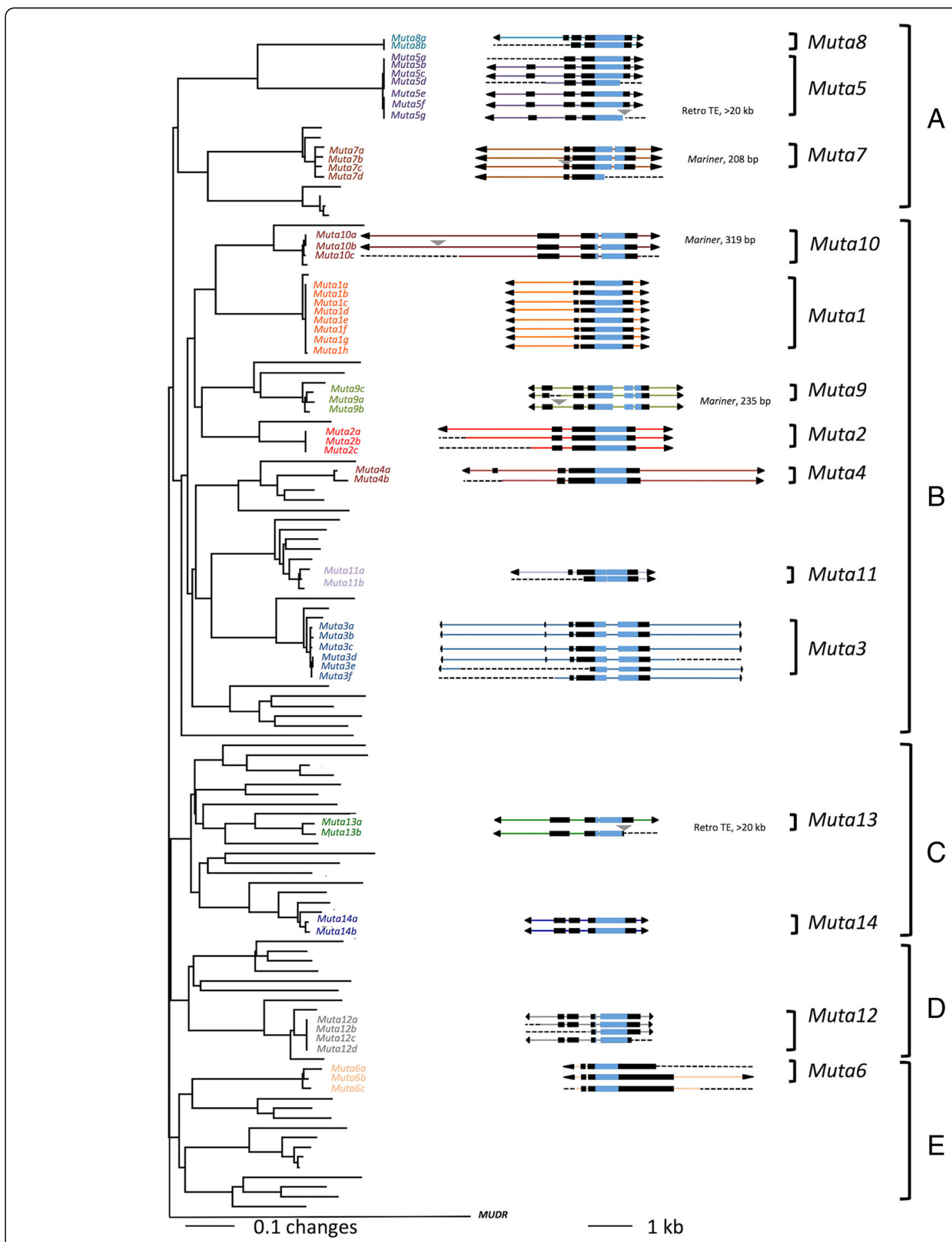

Fig. 1 Phylogenetic tree and structure of MULEs in Ae. aegypti. Neighbor-joining tree generated from a multiple alignment of 141 conceptually translated catalytic domains from transposase proteins with bootstrap values calculated from 1000 replicates. For element structures: TIRs are black triangles, exons are black boxes, DDE domains are blue boxes, introns are lines connecting boxes, colored lines indicate within family identity of noncoding regions, other TE insertions are gray triangles above elements, and dashed lines are missing sequences caused by gaps, deletions or large insertions. The maize MuDR transposase is used as an outgroup. The Ae. aegypti Mutator transposases are classified into 5 major lineages a-e 
was detected between these elements. In another example, nucleotide similarity between Muta3 and Muta11 is restricted to the DDE region (71\% identity, 390 bp) and the TIRs (76\% identity, $110 \mathrm{bp}$ ).

After removal of other TE insertions and manual correction of frameshifts caused by small insertions and deletions, the 31 full-length elements were predicted to encode transposase proteins ranging in size from 416 to 554 residues (Additional file 2: Table S1). Comparison of conceptually translated transposases identified conserved regions, other than the DDE domain, that could have functional significance. The transposases of 13 families were predicted to harbor a FLYWCH type zinc finger DNA binding domain in the $\mathrm{N}$-terminus, while Muta6 was predicted to harbor a SWIM type zinc finger DNA binding domain in the C-terminus. Each family contains at least one putative full-length copy in which the coding region is not interrupted by frameshifts or stop codons. Of particular interest to this study, 3 families (Muta1, Muta3 and Muta5) include at least 2 members that are identical or nearly identical ( $>99 \%$ identity). These features indicate recent and possible ongoing activity of multiple MULE families in Ae. aegypti.

\section{Identification of the active Muta 1 family}

Of the 14 families, Muta1 appeared to be the best candidate for an active element. The family contains 7 identical copies and an 8th copy with only two point mutations in predicted noncoding DNA. Muta1 is 3198 bp, flanked by TSDs of $8 \mathrm{bp}$ or $9 \mathrm{bp}$, TIRs of $145 \mathrm{bp}$ comprised of a 10 bp imperfect palindromic terminal motif with the 5 th and 6th nucleotide unpaired, and 9 copies of a 12 bp subterminal tandem repeat separated by 3-4 bp spacers (Fig. 2, open and solid arrows, respectively). Because of the complexity of the TIR structure, Muta1 can be classified as a type2 Foldback TE [34]. The Muta1 transposase is predicted to be 504 -amino acid transposase and encoded by two exons.
Over 300 putative nonautonomous elements derived from Muta1 were detected in the Ae. aegypti genome (e- value $<10^{-10}$ ), with 171 flanked by perfect TSDs of 8 bp or 9 bp (Additional file 3: Figure S2A). Most of the derivative elements share the TIR sequence with Mutal, but the TIRs are often truncated, with variable copies of the subterminal repeats (Fig. 3). There are multiple copies of some derivative elements; for example, there are 4 copies of Muta1NA1 (>98\% identical). Most derivative elements contain variable internal sequences and share sequence similarity with only the TIR of Muta1. For about $20 \%$ of the derivative elements these variable internal regions can be aligned with sequences from host genes, much like previously described Pack-MULEs [11]. For example, the 1623 bp Muta1NA3 contains a 276 bp fragment from a serine/threonine-protein kinase gene $(97.5 \%$ identical, evalue $<1$ e-51) (Fig. 3). Among the 171 insertion sites, 110 $(64.3 \%)$ are located in gene bodies or within $5 \mathrm{~kb}$ upstream or downstream of genes. In a control dataset of 171 randomly selected genomic sites (see methods) only 49 (28.7\%) were located in the same regions (Additional file 3: Figure S2B), suggesting that Muta1 may have an insertion preference for genic regions as was previously reported for plant MULEs [6].

\section{Muta1 can transpose in yeast}

A yeast transposition assay was employed to determine whether Muta1 transposase is able to catalyze the movement of natural and/or artificial nonautonomous elements. In prior studies members of five superfamilies (MULE, Tc1/mariner, hAT, PIF/Harbinger and piggyBac) were shown to transpose in yeast [15, 35-38]. Our yeast assay consisted of two plasmid constructs: an expression vector containing the Mutal transposase coding sequence downstream of the galactose inducible GAL1 promoter, and a reporter vector containing a nonautonomous element inserted in, and blocking expression of, the $A D E 2$ gene (Additional file 4: Figure S3A). We first

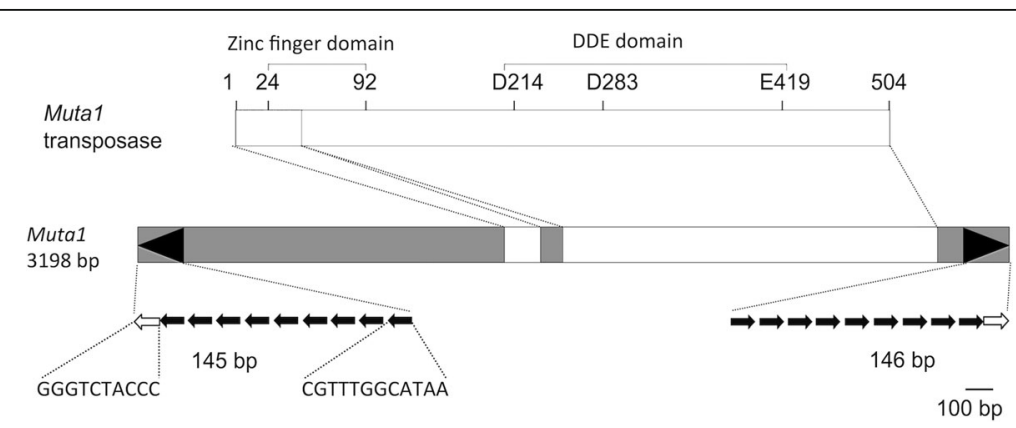

Fig. 2 Structural features of the Mutal element and its transposase. The eight virtually identical Mutal elements contain noncoding regions (shaded) and the coding region (white boxes) for the predicted 504-residue transposase with the predicted zinc and catalytic (DDE) domains discussed in the text. Structural features of Muta1 include its distinctive long TIR (black arrowheads) whose substructure, expanded at the bottom, includes the $10 \mathrm{bp}$ terminal palindromic motif (open arrow) and the 12 bp subterminal tandem repeats (black arrows) with linker DNA of 3-4bp represented by gaps between solid arrows 


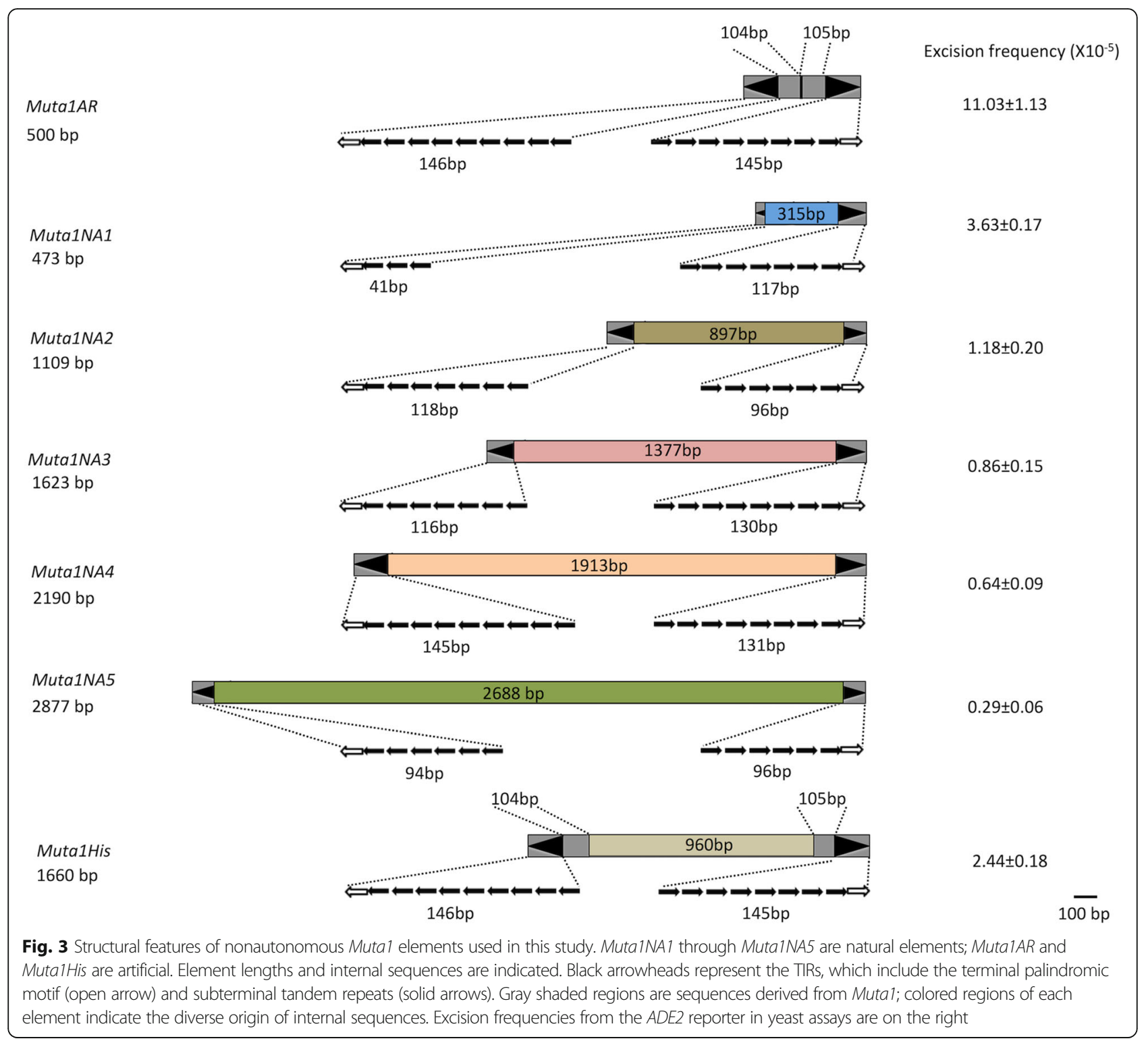

tested the artificial Muta1AR element which contains $250 \mathrm{bp}$ from each end of Muta1 (Fig. 3). Transposase mediated TE excision restored $A D E 2$ expression and resulted in reversion of adenine auxotrophy to prototrophy (Additional file 4: Figure S3B, Additional file 5: Figure S4A). Excision events were validated by PCR amplification of the $A D E 2$ empty site from revertants (Additional file 6: Figure S5A).

In subsequent experiments, the reporter plasmid was modified by inserting several natural nonautonomous elements (Muta1NA1-5, Fig. 3, Additional file 5: Figure S4B-F) into the $A D E 2$ coding region. Despite differences in TIR length and the length and sequence of internal regions, Muta1 transposase was able to mobilize all of these elements, but none with a frequency as high as the artificial element Muta1AR.
Integration events catalyzed by Muta1 transposase were assayed by first constructing the nonautonomous Muta1HIS element containing a yeast selectable marker HIS3 gene flanked by 250 bp of the Muta1 termini (Fig. 3). Integration of Muta1HIS into yeast chromosomes was assayed in plasmid-free cells following selection with 5-FOA, (see Methods, Additional file 4: Figure $\mathrm{S} 3 \mathrm{D})$. Comparison of the frequency of $A D E 2$ revertants retaining the HIS3 marker and are 5-FOA resistant $\left(2.71 \times 10^{-6} \mathrm{His}^{+} 5\right.$-FOA ${ }^{\mathrm{R}}$ cells/total cells $)$ to the frequency of total $A D E 2$ revertants $\left(2.44 \times 10^{-5}\right.$ Ade $^{+}$cells/ total cells) indicated that about $11 \%$ of the Muta1HIS elements excised from the donor plasmid had reintegrated in yeast chromosomes (Table 1). In another assay, $A D E 2$ colonies isolated directly from the Muta1HIS excision assay were tested to determine if they were also 
$\mathrm{His}^{+}$and $5-\mathrm{FOA}^{\mathrm{R}}$. Of 300 revertant colonies, 41 (14\%) could proliferate on selective plates, in agreement with the results of the first approach (Table 1).

To determine the precise insertion sites, polymorphic bands on transposon display gels were recovered [39], sequenced and mapped to yeast chromosomes (Additional file 6: Figure S5B). Sixty of 62 sites had significant matches with yeast genomic sequence, while two matched plasmid sequences. Fourteen of the 60 genomic sites were in gene bodies while 27 were within $1 \mathrm{~kb}$ of genes. Thus $41 / 60$ insertions (68\%) were in gene-rich regions [40]. Amplification and sequencing of each site revealed the presence of TSDs with 8 bp for 21 events and 9 bp for 39 events (Additional file 7: Table S2). Consensus sequences generated from the yeast insertion sites and the 171 sites for Muta1 derivative elements in the Ae. aegypti genome indicated little or no sequence preference for insertion (Additional file 8: Figure S6).

\section{The Muta5 element does not transpose in yeast}

The successful transposition of Muta1 in yeast prompted us to perform a similar analysis of the Muta5 family which contains 3 identical copies, a 4th with $>99 \%$ sequence identity, and 3 copies with large deletions or insertions (Fig. 1). Muta5 is $3496 \mathrm{bp}$, flanked by TIRs of $151 \mathrm{bp}$ and TSDs of $8 \mathrm{bp}$ or $9 \mathrm{bp}$ and is predicted to contain 3 exons that encode a 554-amino acid transposase. The TIR of Muta5 contains 9 copies of a 15 bp subterminal tandem repeat and an 8bp terminal motif (Additional file 9: Figure S7A). There are over 200 Muta5 derivative elements (e- value $<10^{-15}$ ) in the Ae. aegypti genome, with 93 flanked by 8 bp or 9 bp TSD. Taken together the features of the Muta5 family strongly suggested that it was an active element. However, in the yeast transposition assay the Muta5 transposase was unable to catalyze transposition of Muta5AR (Additional file 9: Figure S7A), which contains $250 \mathrm{bp}$ from the ends of Muta5 (Additional file 9: Figure S7B).

Table 1 Muta1 integration in yeast

\begin{tabular}{|c|c|}
\hline Assay & Frequency \\
\hline \multicolumn{2}{|l|}{ MutalHis integration $^{a}$} \\
\hline ade $2^{+}$excisant & $2.44 \times 10^{-5} \mathrm{Ade}^{+}$cells/total cells \\
\hline \multirow[t]{2}{*}{$\mathrm{His}^{+} 5-\mathrm{FOA}^{\mathrm{R}}$ excisant } & $2.71 \times 10^{-6} \mathrm{His}^{+} 5 \mathrm{FOA}^{\mathrm{R}}$ cells/total cells \\
\hline & ratio of reintegration $=11 \%$ \\
\hline \multicolumn{2}{|l|}{ MutalHis integration ${ }^{\mathrm{b}}$} \\
\hline ade $^{+}$excisant & 300 colonies \\
\hline \multirow[t]{2}{*}{$\mathrm{His}^{+}$5-FOA ${ }^{\mathrm{R}}$ excisant } & 41 colonies \\
\hline & ratio of reintegration $=14 \%$ \\
\hline
\end{tabular}

${ }^{a}$ Measured by selection for presence of Muta1His and absence of plasmid following excision from ADE2

${ }^{\mathrm{b}}$ Measured by analysis of independent $\mathrm{Ade}^{+}$revertants to maintain Muta1His but lacking plasmid

\section{TSD at donor site affects Muta1 transposition in yeast}

Successful transposition of Muta1 in yeast facilitated the analysis of the importance of its features by quantifying the impact of mutations on transposition quality and frequency. With regard to the role of the TSDs, although the nonautonomous constructs used in the assays described thus far lacked flanking TSDs, their reinsertion still generated TSDs of 8 bp or 9 bp. To examine the impact of TSD length or sequence on excision frequency of Muta1AR, three versions of 8 bp (TTCAATAG, CGATTCAA and GGTAACTC) or 9 bp (ATTCAATAG, TCGATTCAA and CGGTAACTC) TSDs were tested. Addition of $8 \mathrm{bp}$ or $9 \mathrm{bp}$ TSDs at the donor site increased Muta1AR excision frequency by $\sim 7$ fold and $\sim 3$ fold, respectively when compared to the controls lacking TSDs (Table 2). Similarly, introduction of TSDs flanking Muta1HIS increased reintegration by about $40 \%$ and $90 \%$ for 8 bp TSDs or 9 bp TSDs respectively (Table 2). For both excision and integration, TSD sequence had little impact.

We next addressed the question of whether the presence or absence of TSDs at the donor site impacts the quality of excision by analyzing so-called transposon footprints. Specifically, class 2 TEs often leaves a footprint upon excision consisting of a few nucleotides or small rearrangements at the site of excision site [41]. Formation of footprints involves DNA repair of sequences flanking the excised element. To assess the impact of TSDs on the repair of excision sites, the donor element construct was modified so that all excision footprints (not only those that maintain the reading frame) could be analyzed. First, nonautonomous elements were inserted in the 5' UTR of $A D E 2$ (Additional file 4: Figure S3C). Second, because insertion of Muta1AR in the 5 'UTR resulted in leaky $A D E 2$ expression, we substituted the longer Muta1NA1 (Fig. 3), which, in the absence of transposase, blocked $A D E 2$ expression. When either 8 bp or 9 bp TSDs were added to Muta1NA1, about $90 \%$ of revertants were precise (Table 2), meaning that the element was removed as well as a single copy of the TSD. The quality of excision appeared to be independent of TSD sequence (Additional file 10: Figure S8B-G). In contrast, absence of donor site TSDs reduced perfect excision to only $10 \%$ of the $A D E 2$ revertant colonies sequenced (Table 2). Most excision sites reflected loss of a few nucleotides from either side of the flanking DNA. Occasionally, part of the TIR (up to $13 \mathrm{bp}$ ) was left after excision and repair (Additional file 10: Figure. S8A).

\section{Mutagenesis of Muta1 transposase}

The catalytic domains of all characterized transposases of class 2 TEs contain a DDE/D amino acid triad [20] and mutagenesis studies confirmed its functional significance in the piggyBac, Mariner and hAT superfamilies [38, 42, 43]. Alignment with the transposase of other 
Table 2 Impact of TSD on transposition

\begin{tabular}{lllll}
\hline $\begin{array}{l}\text { TSD } \\
\text { length }\end{array}$ & $\begin{array}{l}\text { TSD } \\
\text { sequence }\end{array}$ & $\begin{array}{l}\text { Excision } \\
\text { frequency }\left(\times 10^{-5}\right)\end{array}$ & $\begin{array}{l}\text { Reintegration } \\
\text { frequency (\%) }\end{array}$ & $\begin{array}{l}\text { precise excision } \\
\text { /examined excision }\end{array}$ \\
\hline Obp & & $11.03 \pm 1.13$ & $13.9 \pm 1.8$ & $4 / 38$ \\
8bp & TTCAATAG & $63.34 \pm 9.12$ & $20.88 \pm 1.53$ & $25 / 29$ \\
& CGATTCAA & $76.33 \pm 11.53$ & $18.22 \pm 2.90$ & $27 / 31$ \\
& GGTAACTC & $68.53 \pm 12.16$ & $19.15 \pm 2.39$ & $24 / 27$ \\
9bp & ATCAATAG & $31.52 \pm 5.27$ & $27.83 \pm 3.01$ & $27 / 29$ \\
& TCGATTCAA & $36.43 \pm 6.12$ & $24.35 \pm 1.98$ & $28 / 30$ \\
& CGGTAACTC & $28.38 \pm 3.11$ & $29.01 \pm 2.76$ & $26 / 30$ \\
\hline
\end{tabular}

${ }^{\mathrm{a}}$ Measured by analysis of independent $\mathrm{Ade}^{+}$revertants to maintain Muta1His but lacking plasmid

active MULEs showed that the DDE triad in Muta1 corresponds to D214, D283 and E419 (Fig. 4a). To determine if these conserved sites play key roles in transposition, site-directed mutagenesis was performed. Transposition activity was completely abolished when D214, D283 or E419 was mutated to alanine (Fig. 4b, Additional file 5: Figure S3I-K). In contrast, mutation of nonconserved sites, including E129, E188, E239, W313 and D473 to alanine had little impact. Although E373 is not a conserved site, mutation to alanine also completely abolished transposition activity.

The functional significance of two additional highly conserved residues in Muta1, H307 and W401, were tested. In a prior study these residues were shown to be essential for transposition of the $h A T$ superfamily member Hermes [21, 43]. Mutation of the corresponding Hermes $\mathrm{H}$ and $\mathrm{W}$ residues ( $\mathrm{H} 268$ and W319) to alanine completely abolished transposition activity. Similarly for the Muta1 transposase, mutation of H307 or W401 to alanine abolished activity (Fig. 4b). Analysis of Hermes transposase also showed that the W319 residue was likely necessary for the correct positioning of flanking DNA during the excision reaction, and that other aromatic residues can partially substitute for this function [21]. When W401 of Muta1 was mutated to phenylalanine, transposition activity was reduced by $79 \%$ (Fig. 4b,

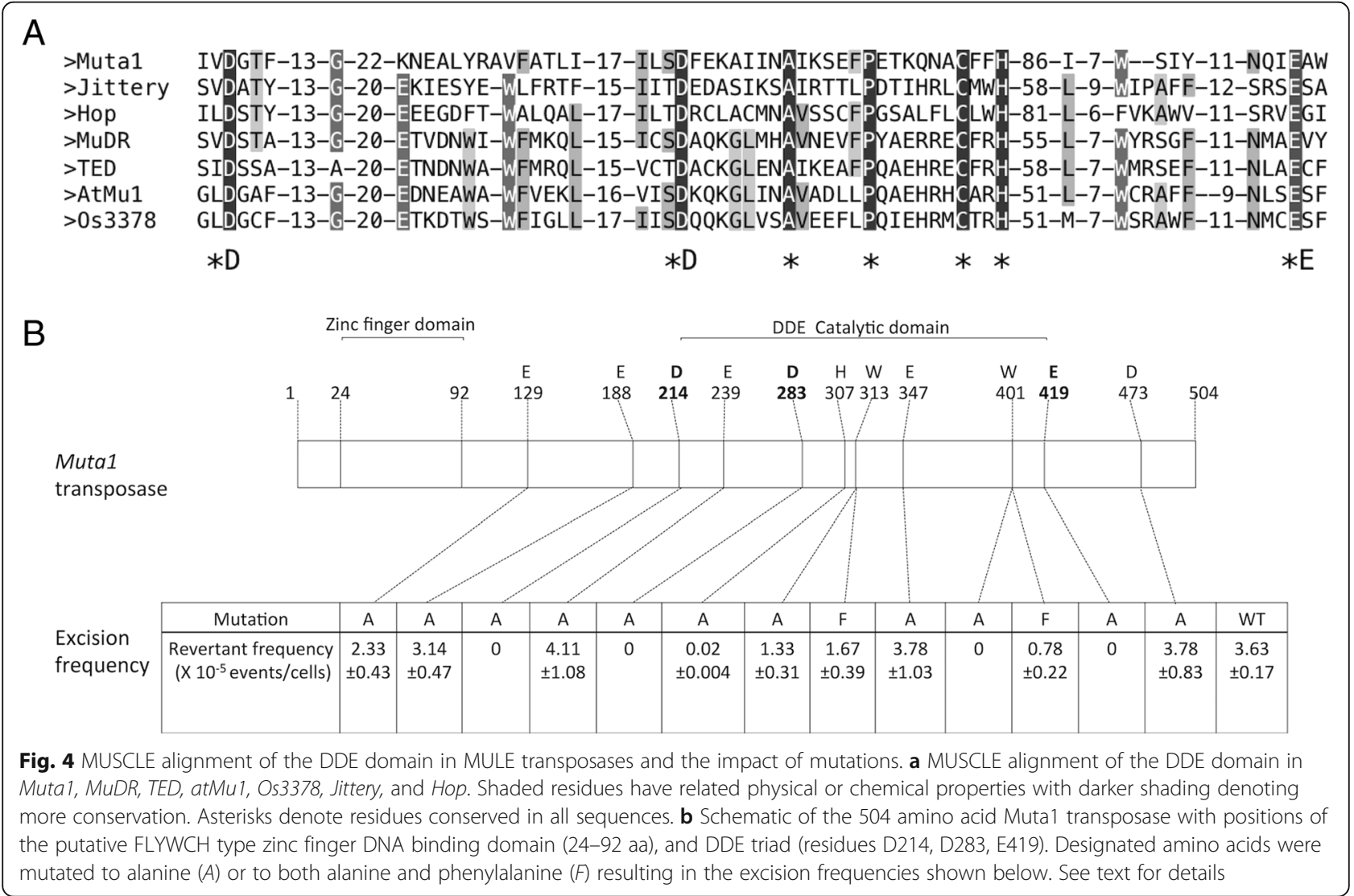


Additional file 5: Figure S3L) and the frequency of precise excision dropped from $90 \%$ to approximately $48 \%$ (8 bp donor site TSD, 14/29 events) and 50\% (9 bp donor site TSD, 15/30 events) (Additional file 10: Figure S8H\&I), suggesting that the Muta1 W401F mutation also led to inaccurate positioning. These results confirmed the importance of the putative DDE motif, the conserved H307, W401 and identified the nonconserved E373 as a potentially important residue for transposase function.

\section{Discussion}

The MULE superfamily is widespread in eukaryotic genomes and is closely related to prokaryotic IS256 elements. However, although it is also found in the genomes of many insects, no active elements have been reported in insect species. In this study, we performed a thorough search for potentially active MULEs in the Ae. aegypti genome and demonstrate that Muta1 encodes a transposase that catalyzes the excision and reinsertion of nonautonomous derivative elements in yeast. With the availability of this heterologous transposition assay, the function of the conserved MULE DDE domain and the role of TSD in transposition were tested.

The DDE/D domain is proposed to be the catalytic core involved in transposition of class 2 TEs and has been identified in all superfamilies [20]. Prior to this study, the functional significance of this domain had been experimentally validated only for members of the piggyBac, Tc1/Mariner, and $h A T$ superfamilies [38, 42, 43]. Results of this study provide the first experimental evidence for the importance of the DDE motif in the transposition reaction of Muta1, a member of the MULE superfamily. Specifically, transposition was completely abolished when any of the three residues were mutated to alanine (Fig. 4b, Additional file 5: Figure S3I-K).

In addition to the DDE triad, other residues critical for transposition were identified. W401 of Muta1 is a conserved residue that is also found in the $h A T$ superfamily $[20,43]$. Crystallographic analysis and in vitro biochemical assays showed that the corresponding W318 residue in Hermes functions in the positioning of flanking DNA, which ensures that the double strand break occurs at the correct position when an element excises from flanking DNA [21]. Other aromatic residues partially substitute for its function, however the mutant transposase generated additional species of intermediates in double strand break repair than the wild-type transposase, suggesting that inaccuracy in the position of the cleavage site may be the cause by these mutations [21]. For Muta1, the W401A mutation completely abolished transposition activity while the W401F mutation resulted in a $79 \%$ reduction of transposition frequency (Fig. 4b, Additional file 5: Figure S3L) and caused inaccurate excision as the frequency of precise excision dropped from $90 \%$ to $~ 50 \%$
(Additional file 10: Figure S8H\&I). Taken together these data suggest that this conserved tryptophan residue is likely playing a similar role in $h A T$ and MULE transposases, which is to correctly position flanking DNA for the excision reaction. In addition to the $\mathrm{W}$ residue, a $\mathrm{CxxH}$ motif is also shared between MULE and $h A T$ elements, the Hermes H268 was found to be located close to the DDE active center and involved in the interaction with TIRs [21]. Mutation of the corresponding H307 to alanine resulted in a $99.5 \%$ reduction of Muta1 transposition activity (Fig. 4b), suggesting the importance of this residue for Muta1 transposase function. Taken together, our study provides experimental evidence to support the close evolutional relationship reported previously between the MULE and $h A T$ superfamilies [20].

Prior to this study, the only MULE shown to transpose in a heterologous host was the rice Os3378 element [15]. Because both Muta1 and Os3378 have demonstrated activity in yeast assays that employed very similar experimental design and nonautonomous elements of similar size, comparison of assay results may be informative (Table 3). Excision frequencies of the $500 \mathrm{bp} \mathrm{Muta1AR,}$ as high as 6940 events per $10^{7}$ cells (Fig. 2), is $\sim 320$ fold higher than Os3378NA469 (469bp). For both elements, excision frequencies are increased by the presence of donor site TSD with Muta1AR enhanced by $\sim 7$ fold ( 8 bp) and 3 fold (9 bp), and Os3378NA469 enhanced by $\sim 17$ fold with TSDs of 9 bp (Table 3). About $80 \%$ of Os3378 integration sites in the yeast genome were located in gene bodies or within $1 \mathrm{~kb}$ of flanking regions of genes while Muta1 had a slightly lower ratio of $68 \%$. In summary Muta1 shows very similar transposition behavior as Os3378, and the higher activity of Muta1 makes it a better tool for the future study of MULE transposition, for example, the biochemical process of excision and integration, and how Pack-MULEs capture host gene fragments [11].

The presence of donor site TSDs impacts the quality of Muta1-mediated excision events as various footprints

Table 3 Comparison of Muta1 and Os3378 transposition in yeast

\begin{tabular}{lll}
\hline & Muta1 & OS3378 \\
\hline Excision frequency no TSD $\left(\times 10^{-7}\right)^{\mathrm{a}}$ & 1103 & 1.2 \\
Excision frequency 8bp TSD $\left(\times 10^{-7}\right)^{\mathrm{a}}$ & 6940 & - \\
Excision frequency 9bp TSD $\left(\times 10^{-7}\right)^{\mathrm{a}}$ & 3211 & 20.2 \\
Reintegration frequency No TSD & $13.90 \%$ & $59.26 \%$ \\
Reintegration frequency 8bp TSD & $19.41 \%$ & - \\
Reintegration frequency 9bp TSD & $27.06 \%$ & $39.28 \%$ \\
Percentage of reinsertion in gene rich regions & $80 \%$ & $68 \%$
\end{tabular}

${ }^{\mathrm{a}}$ Based on the excision of Muta1AR or Os3378NA469 from the coding region of $A D E 2$

${ }^{\mathrm{b}}$ Based on the reintegration of Muta1His or Os3378NA469

- Corresponding experiment was not reported 
were generated without donor TSD (Additional file 10: Figure S8A). The predominance of small deletions (1-4 bp) suggests that the Muta1 transposase cuts outside the TIR. In contrast, with the presence of either 8 bp or $9 \mathrm{bp}$ TSD at the donor site, most excision events were precise and the actual TSD sequence did not seem to matter (Additional file 10: Figure S8 B-G). Similar behavior was also observed for IS256, the prokaryotic TE family related to MULEs, and for the one other MULE tested, Os3378. Reduction of TSD from 8bp to 6bp eliminated precise excision of IS256 and reduced the Os3378 precise excision frequency from $97.44 \%$ to $82.05 \%[15,19]$. For IS256 it was hypothesized that precise excision is achieved through a transposase-independent replication slippage mechanism that requires a short stretch of homologous DNA with a minimum length of 8 bp [44]. In our assay, the absence of donor TSDs resulted in a 90\% reduction in precise excision (Additional file 10: Figure S8A-G), which suggests a role for TSDs in promoting precise excision.

Thirty-one full-length MULEs that group into 14 families were identified in the Ae. aegypti genome. Several families have identical or nearly identical full-length copies including Muta1 (7 identical), Muta5 (3 identical), and Muta3 (2 with only 2 noncoding SNPs). Although the existence of identical genomic copies is a feature of active TEs, Muta5 was unable to catalyze the movement of nonautonomous derivative elements in yeast (Additional file 9: Figure S7). One explanation for our success with Muta1 but failure with Muta5 is that the latter has 3 predicted exons while the former has 2 . More predicted exons would increase the chances of incorrectly assembling the actual/functional Muta5 transposase.

Accumulation of seven identical copies of Muta1 in Ae. aegypti suggests that this element may still be active or that it has some success evading the genome surveillance system shown previously to effectively silence exogenous TEs [26-28]. In this regard, it may be possible to engineer Muta1 to make it an effective endogenous mutagen. Like the $M u D R$ system in maize, where the genome has numerous copies of nonautonomous $M u$ elements, there are over 300 Muta1 derivative elements in the Ae. aegypti genome (Additional file 3: Figure S2A). Although mobility in the yeast assay does not guarantee mobility in the host, if Muta1 was able to mobilize even a subset of these elements as it does in yeast, it could be an effective tool for high frequency insertional mutagenesis, especially when coupled with its preference for genic insertions and a lack of target sequence preference (Additional file 3: Figure S2B, Additional file 8: Figure S6).

\section{Conclusions}

This is the first report of the transposition of a nonplant MULE, Muta1, in a heterologous system and provides the first experimental evidence for the functional significance of the DDE domain in the transposition reaction in the MULE superfamily. High frequency transposition in a yeast assay facilitated the determination of Muta1 transposition features including precise excision, genic targeting with no sequence preference and the impact of TIR and TSD for insertion and excision. Taken together, Muta1 may be a valuable tool for forward genetics in mosquitoes.

\section{Methods}

\section{Identification of MULEs in Ae. aegypti}

The mosquito genomes used in this study: AaegL3 build for Ae. aegypti (https://www.vectorbase.org/organisms/ aedes-aegypti/liverpool/aaegl3); AaloF1 build for $A e$. albopictus (https://www.vectorbase.org/organisms/aedesalbopictus/foshan/aalof1); CpipJ2 build for C. quinquefasciatus (https://www.vectorbase.org/organisms/culexquinquefasciatus/johannesburg/cpipj2) and AgamP4 build for An. gambiae (https://www.vectorbase.org/organisms/ anopheles-gambiae/pest/agamp4). The conserved MULE DDE domain from all eukaryotes [20] was used as query to search the mosquito genomes by TBLASTN, as implemented in the TARGeT pipeline [33] with an E-value cutoff of 0.001. Flanking DNA sequences with $10 \mathrm{~kb}$ upstream and downstream of the matched region were retrieved. The ends of a putative element were determined by aligning two closely related elements with their $20 \mathrm{~kb}$ flanking sequences, TIR boundaries and TSDs were manually identified. Coding capacity of each element was predicted by the GENSCAN program (http://genes.mit.edu/GENSCANinfo.html).

To identify Muta1 derivative nonautonomous elements, $50 \mathrm{bp}$ from each end of Muta1 was used in a BLASTN search with TARGeT [33] using default parameters. One hundred bp of flanking DNA sequences were retrieved for manual verification of the TIR and TSD of each derivative element. Fifty bp flanking each element were used for BLASTN searches against the Ae. aegypti genome (AaegL3 build) to determine the genomic location and compared to the genome annotation (release AaegL3.3, https://www.vectorbase.org/organisms/aedesaegypti/liverpool/aaegl3) to determine the adjacent genes. For the control data set, 171 genome coordinates across the 4,757 scaffolds were randomly generated, and compared to the genome annotation (release AaegL3.3) to determine the surrounding sequences and genes. The random insertion sites generation used 1,000 replicates to estimate the expected number of insertions (and standard deviations) in each category.

\section{Yeast construct construction}

Genomic DNA of individual Ae. aegypti mosquito (Liverpool strain, obtained from Dr. Atkinson, UC Riverside) was extracted using the DNeasy Blood \& 
Tissue Kit (Qiagen). The two exons of Muta1 predicted by GENSCAN program were cloned from genomic DNA and fused through overlap PCR (all primer sequences are available in Additional file 11: Table S3). The complete transposase coding sequence was then cloned into the Gateway cloning vector pENTR and transferred to destination vector pGAL415-ccdb [45] with LR Clonase (Invitrogen) to generate the pGAL415-ccdbMutal plasmid (Additional file 3: Figure S2A).

Two hundred fifty bp from each end of Muta1 were fused by overlap PCR to generate Muta1AR. The HIS3 fragment containing the yeast HIS3 coding sequence, HIS3 5 ' and 3' UTR, and HIS3 promoter was cloned from vector pGAL415-ccdb [45]. The HIS3 fragment was then fused with $250 \mathrm{bp}$ from each end of Muta1 through overlap PCR to generate Muta1HIS. Muta1NA1-5 elements were cloned directly from genomic DNA. All nonautonomous elements were inserted in the HpaI site of $A D E 2$ for the exon excision assay or the Xhol site for 5' UTR excision assay through homologous recombination in yeast as previously described [37]. Donor site TSDs were introduced by adding corresponding TSD sequences in primers (Additional file 11: Table S3).

For Muta5 assay, plasmid pGAL415-ccdbMuta5 was constructed in the same way as pGAL415-ccdbMuta1, and Muta5NA was constructed by overlap PCR (Additional file 11: Table S3).

\section{Transposition assay}

The yeast transposition assay using Saccharomyces cerevisiae strain DG2523 and the pWL89a vector was described previously $[35,36]$. Transformation was performed using the Frozen-EZ Yeast Transformation kit (Zymo research). For excision assays, transformants were grown in $5 \mathrm{ml}$ liquid media of CSM -leu-ura with $2 \%$ dextrose. After growth to saturation ( $36 \mathrm{~h}$ ), cells were washed twice with $5 \mathrm{ml}$ water, resuspended in $0.5 \mathrm{ml}$ water and plated onto CSM -his-leu-ade with $2 \%$ galactose. Colonies were counted after incubation at $30^{\circ} \mathrm{C}$ for 15 days and viable counts were made by plating $100 \mu \mathrm{l}$ of a $1 \times 10^{5}$ and $1 \times 10^{6}$ dilution on YPD plates.

For the reintegration assay, cells were grown to saturation in $5 \mathrm{ml}$ liquid CSM -leu-ura with $2 \%$ dextrose, cells were washed twice with $5 \mathrm{ml}$ sterile water, resuspended in $0.5 \mathrm{ml}$ water and plated onto CSM -leu-ura-ade with $2 \%$ galactose plate and CSM -his-leu + 5-FOA with $2 \%$ galactose plates. Colonies were counted after incubation at $30^{\circ} \mathrm{C}$ for 15 days, and viable counts were made by plating $100 \mu \mathrm{l}$ of a $1 \times 10^{5}$ and $1 \times 10^{6}$ dilution on YPD plates. In another approach, individual $\mathrm{Ade}^{+}$Muta1HIS excision revertant colonies isolated directly from plates of CSM -his-leu-ade with $2 \%$ galactose were streaked on CSM -his + 5-FOA plates to calculate the reintegration frequency.

\section{Excision and reinsertion analysis}

For footprint analysis, colony PCR was performed on $A D E 2$ revertant colonies using primers (Table S3) flanking the insertion sites. PCR products were gel extracted (Zymoclean Gel DNA Recovery Kit) and sequenced. For reinsertion analysis, transposon display was conducted [39]. Genomic DNA was extracted from revertant colonies using the Yeastar genomic DNA kit (Zymo research); DNA samples were digested by $B f a$ I followed by adapter ligation. Pre-amplification and selective amplification were used to amplify the sequences between Muta1 TIR and the BfaI adapter sequence. Amplicons consisting of flanking sequences of the reinsertion sites and part of Muta1 TIR were resolved on a $4 \%$ agarose gel, and polymorphic fragments were recovered and sequenced. Flanking sequences were mapped to the yeast genome (S288C, http://yeastgenome.org/) and the reinsertion sites were determined with regard to the closest genomic features. The insertion site analysis figure was made using the program Pictogram http:// genes.mit.edu/pictogram.html.

\section{Mutagenesis of Muta1 transposase}

Site-directed mutagenesis was used to generate mutant versions of Muta1 transposase. One pair of primers (Additional file 11: Table S3) was used for each mutation site, and pGAL415-ccdbMuta1 plasmid was used as template. PCR products were digested with $D p n 1$ to remove template, and the resulting plasmid was sequenced to confirm that mutations occurred as expected.

\section{Additional files}

Additional file 1: Figure S1. Phylogeny and MULE copy number of mosquito and fruit fly species. Phylogenetic relationship and approximate divergence time of Ae.aegypti, Ae. albopictus, C. quinquefasciatu,s and An. gambiae. Genome size and copy number of MULE DDE domain in each species is shown. (TIFF $292 \mathrm{~kb}$ )

Additional file 2: Table S1. Summary of 14 MULE families in Ae. aegypti. (DOCX $81 \mathrm{~kb}$ )

Additional file 3: Figure S2. Features of Mutal derivative elements in Ae. aegypti. (A) 171 Mutal derivative elements divide into 5 groups based on size. Within each group, the number of elements with 8 bp TSD and $9 \mathrm{bp}$ TSD are shown in grey and black, respectively. (B) Distribution of Mutal derivative element insertion sites in the Ae. aegypti genome. Mean \pm s.d., $n=1,000$ (for control). Number of insertion sites in the Ae. aegypti genome and control data set is shown in black and grey, respectively. (TIF $4425 \mathrm{~kb}$ )

Additional file 4: Figure S3. Yeast transposition assay constructs. (A) Structures of pMuta1_PAG415 and pWL89Ae. AmpR, ampicillin resistance gene; ori, E. coli replication origin; Pgal1, GAL1 promoter; CYC1 ter, terminator; CEN, centromere sequences of yeast chromosomes; ARS, autonomous replication site. Dashed lines indicate the position of nonautonomous element insertions, in the 5'UTR and coding region respectively. Black arrows indicate the positions of primers used for PCR analysis in Figure S3A. (B) Excision from coding region of ADE2. (C) Excision from 5' UTR of ADE2. (D) Reintegration. In the parental strain, pWL89A carries Muta1HIS in the coding region of ADE2. Reintegration is assayed by selecting cells that retain the HIS marker in Muta1HIS when 
the parental plasmid is excluded by 5-FOA treatment, which is toxic to Ura $^{+}$cells. (TIF $602 \mathrm{~kb}$ )

Additional file 5: Figure S4. ADE2 revertant colonies from the yeast transposition assay. (A-G) Excision activity of nonautonomous elements from the ADE2 coding region. $(H)$ Negative control, MutalAR excision from the ADE2 coding region is tested on plates without galactose. (I-L) MutaTAR excision from the ADE2 coding region is tested with mutant transposases. (TIF $3512 \mathrm{~kb}$ )

Additional file 6: Figure S5. Analysis of excision and reinsertion events (A) PCR analysis of the Muta 1NA1 excision sites from ADE2 revertants using flanking primers. Expected band size is $820 \mathrm{bp}$ (control) or 350bp, with or without Muta1NA1, respectively. (B) Transposon display analysis of Muta1HIS reinsertion in the yeast genome. DNA bands are amplicons consisting of flanking sequences of the reinsertion sites and part of the TIR. PWL89A-Muta1HIS vector is used as control. Arrows indicate the polymorphic bands that represent the insertion of MutalHIS in different genomic locations. (TIF $413 \mathrm{~kb}$ )

Additional file 7: Table S2. Target Site Duplications (TSDs) and locations of Mutal transpositions in yeast. (DOCX $96 \mathrm{~kb}$ )

Additional file 8: Figure S6. Seqlogo of insertion sites of Ae. aegypti Mutal derivative elements and reintegration sites in yeast. Both 8bp TSDs (A) and 9bp TSDs (B) and their 7bp flanking sequences are analyzed, insertion preference is shown as a pictogram (height of letter indicates percentage of each nucleotide at that position) and the frequencies of preferred nucleotides, if any, are shown. (TIF $1361 \mathrm{~kb}$ )

Additional file 9: Figure S7 Structural features of Muta5 and excision assay results. (A) Structural features of Muta5. White boxes are coding regions, shaded boxes are noncoding regions, and triangles are the TIR. Within the TIR, black arrows represent 9 copies of the 15bp subterminal tandem repeat, open arrow represents the $8 \mathrm{bp}$ terminal motif. The putative 554-residue transposase is predicted to harbor a zinc finger domain and the catalytic (DDE) domain. The artificial Muta5AR element contains 250 bp from each end of Muta5. (B) Excision frequencies of Muta5AR and Muta5AR from the ADE2 reporter in the yeast assay. Muta1 serves as the positive control. (TIF $373 \mathrm{~kb}$ )

Additional file 10: Figure S8. Footprints from Muta1NA1 excision events. Arrows indicate the Muta1NA1 insertion, length and sequence of the TSD in each assay (shown above the arrows). TSD or sequences derived from TSDs are in bold, sequences derived from Muta1NA1 are underlined, the number of recovered events is on the right. (A) Footprints of Muta1NA1 excision from the ADE2 $5^{\prime}$ UTR without donor site TSD. (B-D) Footprints of Muta1NA1 excision from the ADE2 $5^{\prime}$ UTR with different 8 bp TSD sequences. (B) TTCAATAG; (C) CGATTCAA; (D) GGTAACTC. (E-G) Footprints of Muta1NA1 excision from the ADE2 $5^{\prime}$ UTR with different 9 bp TSD sequences. (E) TCGATTCAA, (F) CGGTAACTC, (G) ATTCAATAG. (H-I) Footprints of MutalNA1 excision from the ADE2 5'UTR with the transposase W401F mutation. (H) the 8bp TSD TTCAATAG was used. (I) the 9bp TSD ATTGAATAG was used. (TIF $665 \mathrm{~kb}$ )

Additional file 11: Table S3. Primers used in this study. (DOCX 124 kb)

\section{Abbreviations}

MULE: Mutator-like transposable elements; PCR: Polymerase chain reaction; TE: Transposable element; TIR: Terminal inverted repeat; TSD: Target site duplication

\section{Acknowledgements}

We thank Jim Burnette, Lu Lu, Brad Cavinder, Peter Atkinson and Jason Stajich for their technical assistance and advice.

\section{Funding}

This research funded by a grant from the W.M. Keck Foundation to SRW.

\section{Availability of data and materials}

The materials and datasets in the current study are available from the corresponding author on request.

\section{Authors' contributions}

$\mathrm{KL}$ performed the sequence analysis, designed and carried out the yeast vector construction and transposition assays. SRW conceived the study, participated in its design and coordination and helped to draft the manuscript. Both authors read and approved the final manuscript.

\section{Competing interests}

The authors declare that they have no competing interests.

\section{Ethics approval and consent to participate}

Not applicable.

\section{Consent for publication}

Not applicable.

\section{Author details}

${ }^{1}$ Graduate Program in Botany and Plant Sciences, University of California, Riverside, CA 92521, USA. 'Department of Botany and Plant Sciences,

University of California, Riverside, CA 92521, USA

Received: 21 October 2016 Accepted: 19 December 2016

Published online: 11 January 2017

\section{References}

1. Finnegan DJ. Eukaryotic transposable elements and genome evolution. Trends Genet. 1989:5:103-7.

2. Wicker T, Sabo F, Hua-Van A, Bennetzen JL, Capy P, Chalhoub B, Flavell A, Leroy P, Morgante M, Panaid O, Paux E, SanMiguel P, Schulman AH. A unified classification system for eukaryotic transposable elements. Nat Rev Genet. 2007;8:973-82.

3. Feschotte C, Pritham EJ. DNA transposons and the evolution of eukaryotic genomes. Annu Rev Genet. 2007;41:331-68.

4. Bao W, Jurka MG, Kapitonov W, Jurka J. New Superfamilies of Eukaryotic DNA Transposons and Their Internal Divisions. Mol Biol Evol. 2009;26(5):983-93.

5. Robertson DS. Characterization of a mutator system in maize. Mutat Res. 1978:51:21-8.

6. Lisch D. Regulation of the Mutator System of Transposons in Maize Methods Mol Biol. 2013;1057:123-42.

7. Neuveglise C, Chalvet F, Wincker P, Gailardin C, Casaregola S. Mutator-like element in the yeast Yarrowia lipolytica displays multiple alternative splicings. Eukaryot Cell. 2005;4:615-24.

8. Pritham EJ, Feschotte C, Wessler SR. Un-expected diversity and differential success of DNA transposons in four species of entamoeba protozoans. Mol Biol Evoanl. 2005:22:1751-63.

9. Lopes FR, Silva JC, Benchimol M, Costa GG, Pereira GA, Carareto CM. The protist Trichomonas vaginalis harbors multiple lineages of transcriptionally active Mutator-like elements. BMC Genomics. 2009;10:330.

10. Lisch D. Mutator transposons. Trends Plant Sci. 2002;7:498-504.

11. Jiang N, Bao Z, Zhang X, Eddy SR, Wessler SR. Pack-MULE transposable elements mediate gene evolution in plants. Nature. 2004:431:569-73.

12. Chalvet F, Grimaldi C, Kaper F, Langin T, Daboussi MJ. Hop, an active Mutator-like element in the genome of the fungus Fusarium oxysporum. Mol Biol Evol. 2003:20:1362-75.

13. Xu Z, Yan X, Maurais S, Fu H, O'Brien DG, Mottinger J, Dooner HK. Jittery, a Mutator distant relative with a paradoxical mobile behavior: Excision without reinsertion. Plant Cell. 2004;16:1105-14.

14. Li Y, Harris L, Dooner HK. TED, an autonomous and rare maize transposon of the Mutator superfamily with a high gametophytic excision frequency. Plant Cell. 2013;25:3251-65.

15. Zhao D, Ferguson A, Jiang N. Transposition of a rice Mutator-like element in the yeast Saccharomyces cerevisiae. Plant Cell. 2015;27:132-48.

16. Babu MM, Iyer LM, Balaji S, Aravind L. The natural history of the WRKYGCM1 zinc fingers and the relationship between transcription factors and transposons. Nucleic Acids Res. 2006:34(22):6505-20.

17. Nesmelova IV, Hackett PB. DDE transposases: Structural similarity and diversity. Adv Drug Deliv Rev. 2010;62(12):1187-95.

18. Eisen JA, Benito MI, Walbot V. Sequence similarity of putative transposases links the maize Mutator autonomous element and a group of bacterial insertion sequences. Nucleic Acids Res. 1994;22:2634-6. 
19. Loessner I, Dietrich K, Dittrich D, Hacker J, Ziebuhr W. Transposasedependent formation of circular IS256 derivatives in Staphylococcus epidermidis and Staphylococcus aureus. J Bacteriol. 2002;184:4709-14.

20. Yuan YW, Wessler SR. The catalytic domain of all eukaryotic cut-and-paste transposase superfamilies. Proc Natl Acad Sci U S A. 2011;108:7884-9.

21. Hickman AB, Ewis HE, Li X, Knapp JA, Laver T, Doss AL, Tolun G, Steven AC, Grishaev A, Bax A, Atkinson PW, Craig NL, Dyda F. Structural basis of hAT transposon end recognition by Hermes, an octameric DNA transposase from Musca domestica. Cell. 2014;158:353-67.

22. Calisher CH. Persistent Emergence of Dengue. Emerg Infect Dis. 2005;11:738-9.

23. Marchette NJ, Garcia R, Rudnick A. Isolation of Zika virus from Aedes aegypti mosquitoes in Malaysia. Am J Trop Med Hyg. 1969;18:411-5.

24. Aviles G, Sabattini MS, Mitchell CJ. Peroral susceptibility of Aedes albifasciatus and Culex pipiens complex mosquitoes (Diptera: Culicidae) from Argentina to western equine encephalitis virus. Rev Saude Publica. 1990; 24(4):265-9.

25. Lindsay SW, Wilkins HA, Zieler HA, Daly RJ, Petrarca V, Byass P. Ability of Anopheles gambiae mosquitoes to transmit malaria during the dry and wet seasons in an area of irrigated rice cultivation in The Gambia. J Trop Med Hyg. 1991;94(5):313-24.

26. Fraser MJ. Insect Transgenesis: Current Applications and Future Prospects. Annu Rev Entomol. 2012;57:267-89.

27. O'Brochta DA, Sethuraman N, Wilson R, Hice RH, Pinkerton AC, Levesque CS, Bideshi DK, Jasinskiene N, Coates CJ, James AA, Lehane MJ, Atkinson PW. Gene vector and transposable element behavior in mosquitoes. J Exp Biol. 2003;206:3823-34.

28. Scali C, Nolan T, Sharakhov I, Sharakhova M, Crisanti A, Catteruccia F. Postintegration behavior of a Minos transposon in the malaria mosquito Anopheles stephensi. Mol Genet Genomics. 2007;278:575-84.

29. Nene $V$, et al. Genome sequence of Aedes aegypti, a major arbovirus vector. Science. 2007;316:1718-23.

30. Chen XG, et al. Genome sequence of the Asian Tiger mosquito, Aedes albopictus, reveals insights into its biology, genetics, and evolution. Proc Natl Acad Sci U S A. 2015;112:5907-15.

31. Arensburger P, Megy K, Waterhouse RM, Abrudan J, Amedeo P, Antelo B, Bartholomay L, Bidwell S, Caler E, Camara F. Sequencing of Culex quinquefasciatus establishes a platform for mosquito comparative genomics. Science. 2010;330:86-8.

32. Holt RA, et al. The genome sequence of the malaria mosquito Anopheles gambiae. Science. 2002;298:129-49.

33. Han YJ, Burnette JM, Wessler SR. TARGeT: A web-based pipeline for retrieving and characterizing gene and transposable element families from genomic sequences. Nucleic Acids Res. 2009;37, e78.

34. Bingham PE, Zachar Z. Retrotransposons and the FB transposon from Drosophila melanogaster. Mobile DNA Washington D.C: American Society for Microbiology Press. 1989;485-502.

35. Yang $G$, Weil CF, Wessler SR. A rice TC1/mariner-like element transposes in yeast. Plant Cell. 2006;18:2469-78.

36. Weil CF, Kunze R. Transposition of maize Ac/Ds transposable elements in the yeast Saccharomyces cerevisiae. Nat Genet. 2000;26:187-90.

37. Hancock CN, Zhang F, Wessler SR. Transposition of the Tourist-MITE mPing in yeast: an assay that retains key features of catalysis by the class 2 PIF/ Harbinger superfamily. Mob DNA. 2010;1:5.

38. Mitra R, Fain-Thornton J, Craig NL. piggyBac can bypass DNA synthesis during cut and paste transposition. Embo J. 2008;27:1097-109.

39. Biedler J, Qi Y, Holligan D, Della Torre A, Wessler SR, Tu Z. Transposable element (TE) display and rapid detection of TE insertion polymorphism in the Anopheles gambiae species complex. Insect Mol Biol. 2003;12(3):211-6.

40. Lynch M, Sung W, Morris K, Coffey N, Landry CR, Dopman EB, Dickinson WJ, Okamoto K, Kulkarni S, Hartl DL, Thomas WK. A genome-wide view of the spectrum of spontaneous mutations in yeast. Proc Natl Acad Sci U S A. 2008;8105(27):9272-7.

41. Sutton WD, Gerlach WL, Schwartz D, Peacock WJ. Molecular analysis of Ds controlling element mutations at the Adhl locus of maize. Science. 1983;223:1265-8.

42. Brillet B, Bigot Y, Auge-Gouillou C. Assembly of the TC1 and mariner transposition initiation complexes depends on the origins of their transposase DNA binding domains. Genetica. 2007;130(2):105-20.

43. Zhou L, Mitra R, Atkinson PW, Hickman AB, Dyda F, Craig NL. Transposition of $h A T$ elements links transposable elements and V(D)J recombination. Nature. 2004;432:995-1001.
44. Hennig S, Ziebuhr W. A transposase-independent mechanism gives rise to precise excision of IS256 from insertion sites in Staphylococcus epidermidis. J Bacteriol. 2008;190:1488-90.

45. Alberti S, Gitler AD, Lindquist S. A suite of gateway (R) cloning vectors for high-throughput genetic analysis in Saccharomyces cerevisiae. Yeast. 2007;24:913-9.

\section{Submit your next manuscript to BioMed Central and we will help you at every step:}

- We accept pre-submission inquiries

- Our selector tool helps you to find the most relevant journal

- We provide round the clock customer support

- Convenient online submission

- Thorough peer review

- Inclusion in PubMed and all major indexing services

- Maximum visibility for your research

Submit your manuscript at www.biomedcentral.com/submit
Biomed Central 